\title{
Melatonin could offer protection against testicular damage caused by a high-fat diet
}

Researchers from the Anhui Medical University, China, have shown that melatonin can prevent testicular damage caused by hyperlipidaemia-a condition that commonly arises from a high-fat diet and sedentary lifestyle. Zhang and colleagues compared the testicular structures of ApoE wild-type mice fed a normal diet with those of ApoE knockout mice fed a high-fat diet with and without melatonin supplementation.

Mice fed a fatty diet with no melatonin exhibited wrinkling and partial thickening of seminiferous tubule basement membranes, as well as vacuolar degeneration of mitochondria and dilation of endoplasmic reticulum. Significant reductions in the number of mitochondria and lipid droplets in Leydig and Sertoli cells and increases in the number of apoptopic germ cells were also observed.

By contrast, the testicular histostructures of ApoE knockout mice fed a high-fat diet with melatonin supplements were similar to those of the control group.

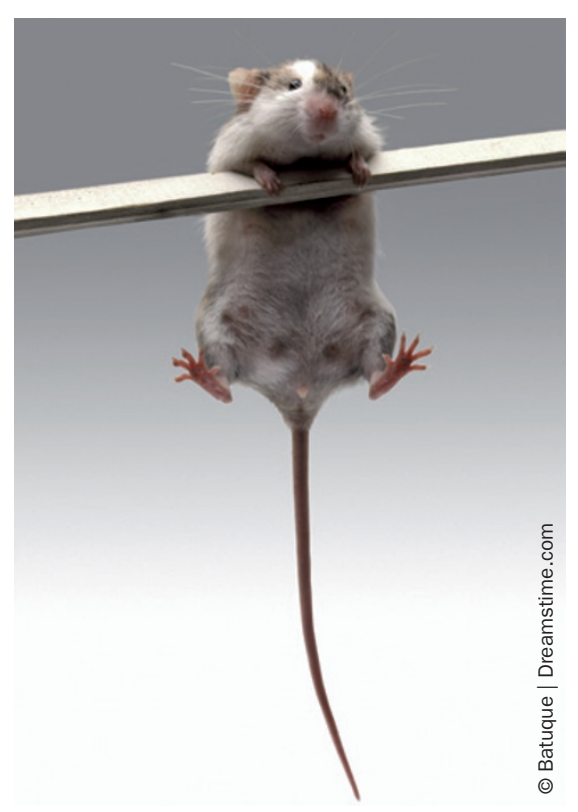

Melatonin is thought to improve lipid profiles via its powerful antioxidant activity and regulatory role in cholesterol metabolism. Referred to as the 'hormone of darkness', it is secreted in the dark and helps to regulate the sleep cycle. Although yet to be approved by the FDA, over-thecounter melatonin-based sleep aids have been available in the USA for years.

In clinical trials, melatonin has also shown promise for the treatment of cancer, cardiovascular diseases, depression, seasonal affective disorder, and sexual dysfunction. This study suggests that trials investigating fertility benefits in hyperlipidaemic men could be next.

Melanie Clyne

Original article Zhang, K. et al. Melatonin prevents testicular damage in hyperlipidaemic mice. Andrologia doi:10.1111/j.1439-0272.2012.01272.x 